\title{
Isolated intermittent vertigo: A presenting feature of persistent trigeminal artery
}

\author{
Rajsrinivas Parthasarathy, Carol Derksen ${ }^{1}$, Maher Saqqur ${ }^{1}$, Khurshid Khan ${ }^{1}$ \\ Department of Neurointerventional Surgery, Institute of Neurosciences, Medanta - The Medicity, Gurgaon, Haryana, India, ${ }^{1}$ Department of Medicine, \\ University of Alberta, Edmonton, Alberta, Canada
}

\begin{abstract}
Embryonic carotid - basilar anastomosis when persistent in adult life can present with a variety of neurological symptoms. We present a patient with isolated intermittent vertigo attributable to the embryonic anastomosis and describe the different types of persistent trigeminal artery. A 76-year-old Caucasian man presented with isolated intermittent vertigo and symptoms suggestive of anterior and posterior circulation strokes. Impaired vasomotor reactivity was demonstrated on insonation of the anterior and posterior cerebral arteries in this patient with a persistent left trigeminal artery and $75 \%$ stenosis of the left internal carotid artery (ICA). The symptom of intermittent vertigo resolved with carotid endarterectomy. Decreased flow across the stenotic segment of the ICA which subserved the posterior circulation resulted in basilar insufficiency. Hypoperfusion to the flocculonodular lobe supplied by the anterior inferior cerebellar artery is a likely cause for the intermittent vertigo.
\end{abstract}

Key words: Hypoperfusion, isolated vertigo, persistent trigeminal artery

\section{Introduction}

A number of embryonic vascular channels connect the developing internal carotid artery (ICA) and the paired longitudinal neural arteries. The embryonic anastomoses primarily serve to feed the paired longitudinal arteries in early embryonic life. The trigeminal artery is the most cephalad of them and is the last to regress. By 7-14 mm embryonic stage, all the embryonic anastomosis disappears and the cervical inter-segmental arteries act to feed the developing basilar artery. ${ }^{[1,2]}$ Though not common, knowledge of these embryonic vessels plays an important role in understanding the mechanisms underlying the various neurological presentations when they persist in adult life.

Address for correspondence:

Dr. Khurshid Khan, 2E3 WC Mackenzie Health Science Center, Edmonton, Alberta T6G 2R7, Canada.

E-mail: khurshid.khan@ualberta.ca

\begin{tabular}{|l|l|}
\hline \multicolumn{2}{|c|}{ Access this article online } \\
\hline Quick Response Code: & Website: \\
\hline & www.ruralneuropractice.com \\
\cline { 2 - 2 } & \\
\hline & \\
\hline
\end{tabular}

\section{Case Report}

A 76-year-old Caucasian male presented with a history of intermittent dizziness. His initial symptom of intermittent short lasting spinning sensation started 6 weeks prior to admission. The vertigo was not positional and not associated with diplopia, dysarthria, or hearing loss. He later experienced word finding difficulty, unsteadiness on his feet and incoordination of the right arm, and blurred vision that affected the right side of his visual field for 3-, 2-, and 1-week prior to admission, respectively. Intermittent vertigo occurred independent of his other symptoms. He is an ex-smoker (7.5 pack years) and took rosuvastatin for dyslipidemia.

On examination, he had mild dysmetria and intentional tremor affecting the right arm, tandem gait ataxia, and right-sided hemianopia. The Dix-Halpike maneuver and head impulse test were negative.

This is an open access article distributed under the terms of the Creative Commons Attribution-NonCommercial-ShareAlike 3.0 License, which allows others to remix, tweak, and build upon the work non-commercially, as long as the author is credited and the new creations are licensed under the identical terms.

For reprints contact: reprints@medknow.com

How to cite this article: Parthasarathy R, Derksen C, Saqqur M, Khan K. Isolated intermittent vertigo: A presenting feature of persistent trigeminal artery. J Neurosci Rural Pract 2016;7:161-3. 
The magnetic resonance imaging of the brain revealed acute diffusion-weighted imaging (DWI) restricted lesion in the external border-zone territory between the middle cerebral artery (MCA) and posterior cerebral artery (PCA), as well as internal border-zone infarct, between the perforators and the cortical branches of the MCA. In addition, there was a cortical prefrontal gyrus DWI restricted lesion. There was $75 \%$ narrowing at the origin of the ICA along with a persistent trigeminal artery (PTA) on the left. Computed tomography angiography [Figure 1]. The basilar artery below the level of the trigeminal artery was hypoplastic, and the posterior communicating artery (PCoA) was absent.

Transcranial Doppler insonation of the left MCA and left PCA revealed impaired vasomotor reactivity (VMR) on breath holding. The breath holding index was 0.35 and -0.25 on insonating the left MCA and PCA, respectively [Figure 2]. Furthermore, the microembolic signal was identified on insonating the left MCA. The symptom of intermittent vertigo resolved with carotid endarterectomy and has not recurred.

\section{Discussion}

The PTA was initially described by Quain in 1844 in an autopsy case. Later, Sutton demonstrated PTA angiographically in $1950 . .^{[1,2]}$ The incidence of PTA varies between $0.1 \%$ and $0.6 \%{ }^{[1]}$ It is the most cephalad of the embryonic carotid - basilar anastomosis. The PTA originates from the ICA at the proximal part of the cavernous segment. It can take either a lateral (petrosal type) or a medial (sphenoidal type) course to reach the posterior fossa. ${ }^{[2,3]}$ The PTA joins the basilar artery between the superior cerebellar and the anterior inferior cerebellar arteries (AICAs). In Saltzman type I

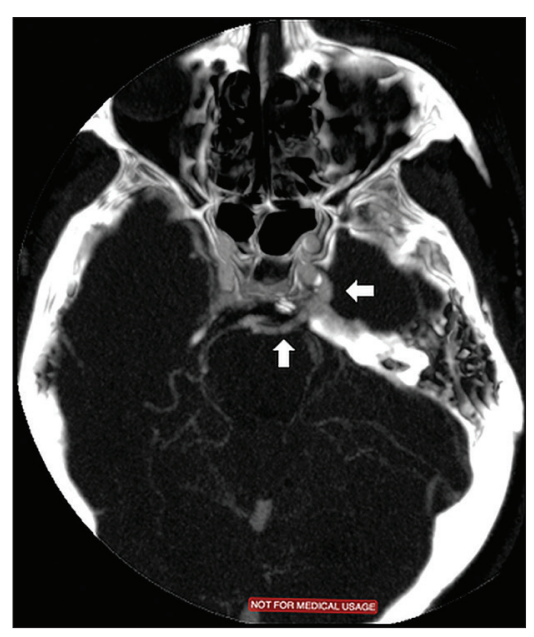

Figure 1: Persistent trigeminal artery on computed tomography angiogram anastomosis, the BA proximal to the PTA and the PCoA are hypoplastic. ${ }^{[2-4]}$ The PTA supplies the upper basilar, superior, and AICA and PCA territories. Conversely, in Saltzman type 2 anastomoses, the PCoA takes over the supply of the PCA territory. The vertebrobasilar system is not hypoplastic, and the posterior fossa structures are supplied by both the PTA and the vertebrobasilar system. In the variant type, PTA supplies one of the ipsilateral cerebellar arteries. Our patient had a Saltzman type 1 PTA.

Posterior circulation stroke due to various etiologies in the context of PTA have been reported in literature and include the following: (a) Cardioembolic occlusion of PTA, (b) ICA dissection, and (c) Large artery atheroembolism. ${ }^{[4-7]}$ Though there have been reports of basilar insufficiency (BI) in patients with PTA and carotid disease, isolated vertigo resulting from impaired VMR has not been objectively demonstrated. ${ }^{[8]}$

Our patient had symptoms of isolated intermittent vertigo, and discrete occurrences suggestive of anterior and posterior circulation ischemic strokes. It is important to note that the symptoms of isolated intermittent vertigo preceded his stroke symptoms by 3 weeks. On imaging, the distribution of strokes in the anterior circulation was in the border-zone territory and frontal cortex suggestive of both a hemodynamic and embolic phenomenon, respectively. Though there was no diffusion restriction, there was a strong clinical suggestion on history and examination that the patient had a posterior circulation stroke. ${ }^{[1]}$ The intermittent vertigo was short, and there were no signs to indicate a peripheral cause for his vertigo. Intermittent vertigo resolved completely with carotid endarterectomy.

Although, the etiology of isolated vertigo is usually peripheral in origin, ischemia involving the flocculonodular lobe supplied by the AICA can present with a similar picture. ${ }^{[11,12]}$ In our patient, the AICA territory is supplied by flow in the PTA. We hypothesize that a preferential involvement of AICA territory due to the following: (a) Altered flow dynamics in the PTA and basilar, (b) Hypoplastic basilar prior to origin of AICA and flow subserved by the PTA, and (c) Probable atheromatous AICA origin ostial stenosis. The intermittent symptoms can be explained by hypoperfusion from BI.

To our knowledge, this is the first case wherein we demonstrated the following: (a) Impaired VMR on insonation of the MCA and PCA on the side of the persistent trigeminal artery, (b) Microembolic signal in the MCA, (c) anterior and posterior circulation strokes, and (d) Isolated vertigo secondary to a hemodynamic phenomenon which resolved with endarterectomy. 


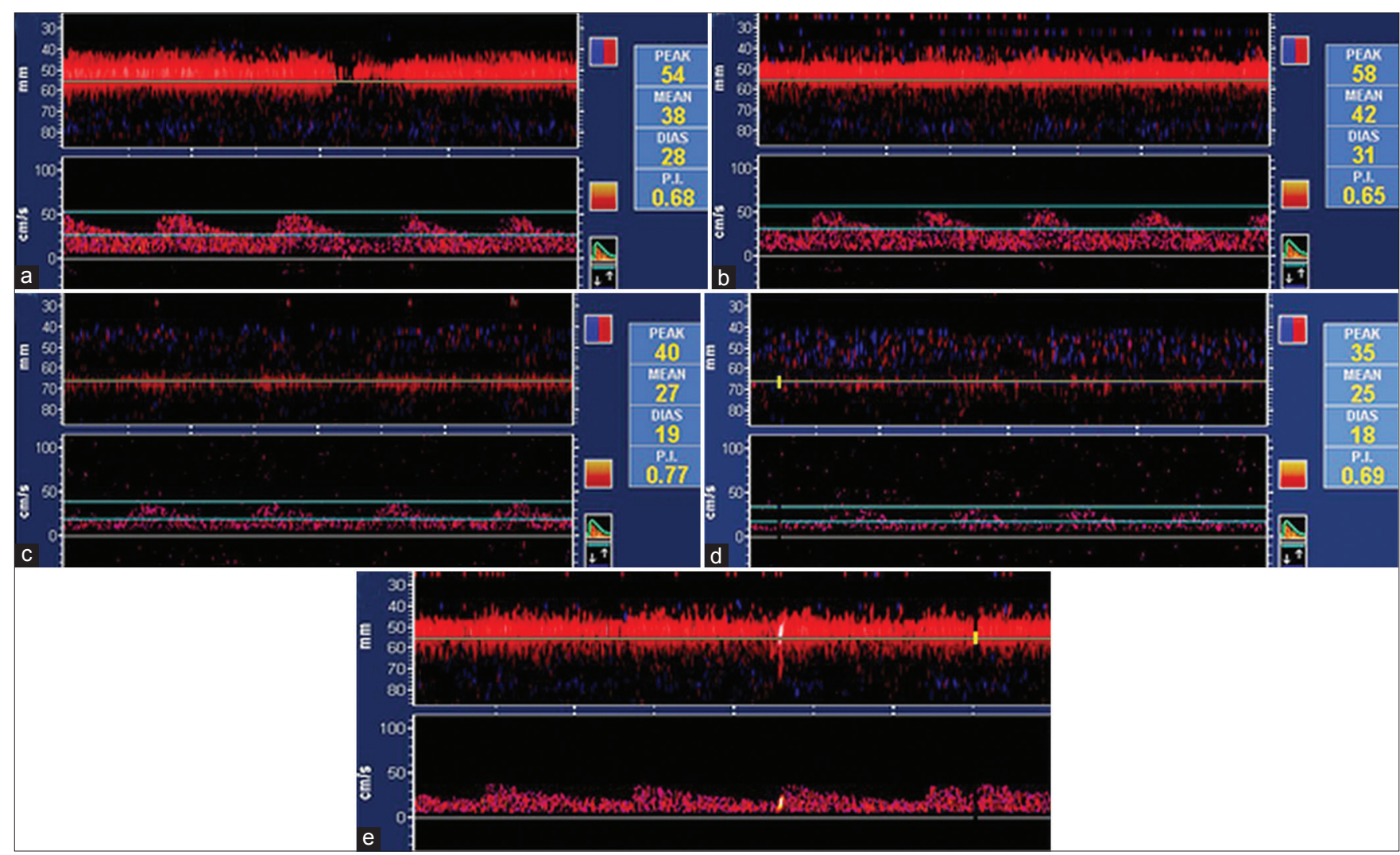

Figure 2: TCD findings on insonation of Left MCA and PCA. (a) Left MCA (baseline) (MFV: 38). (b) Left MCA (end of breath holding) (MFV: 42). (c) Left PCA (baseline) (MFV: 27). (d) Left PCA (end of breath holding) (MFV: 25). (e) MES left MCA. TCD: Transcranial Doppler, MCA: Middle cerebral artery, PCA: Posterior cerebral artery, MES: Microembolic signal, MFV: Mean flow velocity

\section{Conclusion}

Knowledge of the embryonic carotid basilar anastomoses is important to understand uncommon manifestations of the cerebrovascular disease. Isolated intermittent vertigo in most instances is due to a peripheral cause. However, it can rarely be the presenting manifestation of a central cause. Hypoperfusion to the flocculonodular lobe subserved by the AICA is a likely cause for the isolated intermittent vertigo in this patient.

\section{Financial support and sponsorship}

Nil.

\section{Conflicts of interest}

There are no conflicts of interest.

\section{References}

1. Quain R. The Anatomy of the Arteries of the Human Body and Its Applications to Pathology and Operative Surgery. London: Taylor and Walton; 1844. p. 550.

2. Meckel S, Spittau B, McAuliffe W. The persistent trigeminal artery:
Development, imaging anatomy, variants, and associated vascular pathologies. Neuroradiology 2013;55:5-16.

3. Alcalá-Cerra G, Tubbs RS, Niño-Hernández LM. Anatomical features and clinical relevance of a persistent trigeminal artery. Surg Neurol Int 2012;3:111.

4. Raybaud C. Normal and abnormal embryology and development of the intracranial vascular system. Neurosurg Clin N Am 2010;21:399-426.

5. Kwon JY, Lee EJ, Kim JS. Brainstem infarction secondary to persistent trigeminal artery occlusion: Successful treatment with intravenous rt-PA. Eur Neurol 2010;64:311.

6. Iancu D, Anxionnat R, Bracard S. Brainstem infarction in a patient with internal carotid dissection and persistent trigeminal artery: A case report. BMC Med Imaging 2010;10:14.

7. Gasecki AP, Fox AJ, Lebrun LH, Daneault N. Bilateral occipital infarctions associated with carotid stenosis in a patient with persistent trigeminal artery. The Collaborators of the North American Carotid Endarterectomy Trial (NASCET). Stroke 1994;25:1520-3.

8. Hirashima Y, Endo S, Koshu K, Takaku A. Vertebrobasilar insufficiency in a patient with persistent trigeminal artery and stenosis of the ipsilateral carotid bifurcation - case report. Neurol Med Chir (Tokyo) 1988;28:584-7.

9. Wise BL, Palubinskas AJ. Persistent trigeminal artery (carotid-basilar anastomosis). J Neurosurg 1964;21:199-206.

10. Eadie MJ, Jamieson KG, Lennon EA. Persisting carotid-basilar anastomosis. J Neurol Sci 1964;1:501-11.

11. Sylaja PN, Coutts SB, Krol A, Hill MD, Demchuk AM; VISION Study Group. When to expect negative diffusion-weighted images in stroke and transient ischemic attack. Stroke 2008;39:1898-900.

12. Kim HA, Lee H. Recent advances in central acute vestibular syndrome of a vascular cause. J Neurol Sci 2012;321:17-22. 